\title{
INDEPENDENT DISCOVERY OF THE TRANSITING EXOPLANET HAT-P-14b
}

\author{
E. K. Simpson ${ }^{1}$, S. C. C. Barros ${ }^{1}$, D. J. A. Brown ${ }^{2}$, A. Collier Cameron ${ }^{2}$, D. Pollacco ${ }^{1}$, I. Skillen ${ }^{3}$, H. C. Stempels ${ }^{4}$, \\ I. Boisse ${ }^{5}$, F. Faedi ${ }^{1}$, G. Hébrard ${ }^{5}$, J. McCormac ${ }^{1}$, P. Sorensen ${ }^{6}$, R. A. Street ${ }^{7}$, D. Anderson ${ }^{8}$, J. Bento $^{9}$, F. Bouchy $^{5}, 10$, \\ O. W. Butters ${ }^{11}$, B. Enoch ${ }^{2}$, C. A. Haswell ${ }^{12}$, L. HebB ${ }^{13}$, C. Hellier ${ }^{8}$, S. Holmes ${ }^{12}$, K. Horne ${ }^{2}$, F. P. Keenan ${ }^{1}$, \\ T. A. Lister $^{7}$, P. F. L. Maxted ${ }^{8}$, G. R. M. Miller ${ }^{2}$, V. Moulds ${ }^{1}$, C. Moutou ${ }^{14}$, A. J. Norton ${ }^{12}$, N. Parley $^{2}$, A. Santerne $^{14}$, \\ B. Smalley ${ }^{8}$, A. M. S. Smith ${ }^{8}$, I. Todd ${ }^{1}$, C. A. Watson ${ }^{1}$, R. G. West ${ }^{11}$, And P. J. Wheatley ${ }^{9}$ \\ ${ }^{1}$ Astrophysics Research Centre, School of Mathematics and Physics, Queen's University Belfast, Belfast BT7 1NN, UK \\ ${ }^{2}$ SUPA, School of Physics and Astronomy, University of St. Andrews, North Haugh, St. Andrews, Fife KY16 9SS, UK \\ ${ }^{3}$ Isaac Newton Group of Telescopes, Apartado de Correos 321, E-38700 Santa Cruz de la Palma, Tenerife, Spain \\ ${ }^{4}$ Department of Physics and Astronomy, Uppsala University, Box 516, SE-751 20 Uppsala, Sweden \\ ${ }^{5}$ Institut d'Astrophysique de Paris, UMR7095 CNRS, Université Pierre \& Marie Curie, 98bis Bd. Arago, 75014 Paris, France \\ ${ }^{6}$ Nordic Optical Telescope, Apartado de Correos 474, E-387 00 Santa Cruz de la Palma, Canary Islands, Spain \\ ${ }^{7}$ Las Cumbres Observatory Global Telescope Network, 6740 Cortona Drive, Suite 102, Goleta, CA 93117, USA \\ ${ }^{8}$ Astrophysics Group, Keele University, Staffordshire ST5 5BG, UK \\ ${ }^{9}$ Department of Physics, University of Warwick, Coventry CV4 7AL, UK \\ ${ }^{10}$ Observatoire de Haute-Provence, CNRS/OAMP, 04870 St. Michel l'Observatoire, France \\ ${ }^{11}$ Department of Physics and Astronomy, University of Leicester, Leicester LE1 7RH, UK \\ ${ }^{12}$ Department of Physics and Astronomy, The Open University, Milton Keynes MK7 6AA, UK \\ ${ }^{13}$ Department of Physics and Astronomy, Vanderbilt University, Nashville, TN 37235, USA \\ ${ }^{14}$ Laboratoire d'Astrophysique de Marseille, 38 rue Frédéric Joliot-Curie, 13388 Marseille Cedex 13, France \\ Received 2010 September 22; accepted 2011 February 16; published 2011 April 5
}

\begin{abstract}
We present SuperWASP observations of HAT-P-14b, a hot Jupiter discovered by Torres et al. The planet was found independently by the SuperWASP team and named WASP-27b after follow-up observations had secured the discovery, but prior to the publication by Torres et al. Our analysis of HAT-P-14/WASP-27 is in good agreement with the values found by Torres et al. and we provide additional evidence against astronomical false positives. Due to the brightness of the host star, $V_{\mathrm{mag}}=10$, HAT-P-14b is an attractive candidate for further characterization observations. The planet has a high impact parameter and the primary transit is close to grazing. This could readily reveal small deviations in the orbital parameters indicating the presence of a third body in the system, which may be causing the small but significant orbital eccentricity. Our results suggest that the planet may undergo a grazing secondary eclipse. However, even a non-detection would tightly constrain the system parameters.
\end{abstract}

Key words: planetary systems - stars: individual (HAT-P-14, WASP-27, GSC 3086-00152) - techniques: photometric - techniques: spectroscopic

Online-only material: supplemental data (FITS) files (tar.gz)

\section{INTRODUCTION}

There has been a rapid increase in the number of transiting planets discovered each year due to dedicated ground- and space-based surveys: HAT (Bakos et al. 2002), TrES (Alonso et al. 2004), XO (McCullough et al. 2005), WASP (Pollacco et al. 2006), CoRoT (Baglin et al. 2006), and Kepler (Borucki et al. 2010). With the discovery of over 35 new planets published in 2010, which represents more than one-third of the total number of transiting planets known, this trend looks set to continue. By increasing the number of well-characterized transiting planets, we are beginning to be able to test theories of planet formation and evolution on a secure statistical basis. For example, the obliquity of hot Jupiters appears to show that the mechanism by which planets migrate cannot be explained solely by planet-disk interactions (Fabrycky \& Winn 2009; Triaud et al. 2010).

Transiting planets orbiting the brightest stars are extremely valuable because extensive follow-up observations of them can be undertaken. This includes secondary eclipse observations to determine atmospheric properties and refine orbital parameters. In order to find planets orbiting bright stars, a wide field of view must be employed to encompass enough stars to make the detection probability significant. It is therefore inevitable that some areas of the sky will be monitored by multiple groups, and indeed several transiting planets have been discovered independently: WASP-11b (West et al. 2009) $=$ HAT-P-10b (Bakos et al. 2009) and XO-5 (Burke et al. 2008; Pál et al. 2009). The independent analyses generally show good agreement; however, $T_{\text {eff }}$ and the radius ratio between the planet and star of WASP-11/HAT-P-10 stand out as being discrepant to some extent, likely caused by systematic errors (Southworth 2010). Independent discoveries therefore provide an important insight into the presence of systematics which can have a significant effect on planet properties.

Here we describe observations of the transiting planet HAT-P-14b (Torres et al. 2010b, hereafter T10) by the SuperWASP survey. The star was monitored by the SuperWASP station on La Palma and followed up with photometric and spectroscopic observations using the Faulkes Telescope North (FTN), Liverpool Telescope, Observatoire de Haute-Province and Nordic Optical Telescope (NOT). The planet was named WASP-27b on 2009 September 27 after the detection was secured, prior to the release of the T10 paper on 2010 March 10. While the HAT-P-14b designation is to be preferred in recognition of priority of publication, it is important that we retain WASP-27b as a secondary designation for statistical completeness in studies of the physical properties of independently discovered WASP planets. 


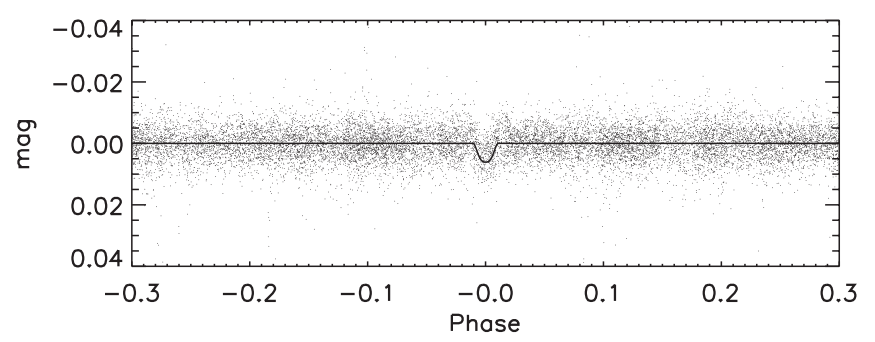

Figure 1. Combined, unbinned SuperWASP light curve for HAT-P-14, folded on the orbital period of $P=4.628$ days. Superimposed is the model transit light curve, based on the system parameters determined from a global fit (see Section 3.2).

(FITS files for this figure are available in the online journal.)

Section 2 describes the observations of HAT-P-14b, including the SuperWASP discovery data and photometric and spectroscopic follow-up. The results of the derived system parameters are presented in Section 3, including an independent spectral analysis. We discuss our findings and compare them to those found by $\mathrm{T} 10$ in Section 4.

\section{OBSERVATIONS}

\subsection{SuperWASP Photometry}

HAT-P-14 is a bright $\left(V_{\mathrm{mag}}=9.98\right)$ star in the constellation Virgo, located at $\alpha_{\mathrm{J} 2000}=17^{\mathrm{h}} 20^{\mathrm{m}} 27^{\mathrm{s}} .87, \delta_{\mathrm{J} 2000}=+38^{\circ} 14^{\prime} 31^{\prime \prime} .9$ (GSC 3086-00152; 2MASS 17202788+3814317). It has been observed by SuperWASP (La Palma) since 2004 and a total of 25,474 photometric points have been collected. A periodic signature was detected in the observations obtained between March and August in 2007, 2008, and 2009. All the data were processed using the pipeline described in Collier Cameron et al. (2006), yielding a detection of a transit-like feature with a period of 4.6278 days in multiple cameras and successive seasons. The folded light curve is shown in Figure 1.

The target underwent several consistency tests aimed at eliminating false positives (see Collier Cameron et al. 2007). Resolved blends were ruled out by inspecting Digitized Sky Survey images which showed the star to be isolated within the WASP photometric aperture. No significant ellipsoidal variability was measurable in the WASP light curve folded on the transit period. The transit depth and duration yielded a planetlike radius for the companion, and a stellar density appropriate to a main-sequence host star of the effective temperature derived from the Two Micron All Sky Survey (2MASS) colors. Having passed all these tests, the star was selected for follow-up observations.

\subsection{Photometric Follow-up}

We obtained six further light curves, shown in Figure 2, in order to refine the photometric parameters. All photometric data presented in this paper are available from the NStED database. ${ }^{15}$

Two full transits were observed with FTN (Hawaii), on 2009 September 18 (SDSS $i$ band) and 2010 May 21 (Pan-STARRS $Z$ filter). The first transit was taken using the Merope camera $\left(\right.$ EM01) ${ }^{16}$ in binning $2 \times 2$ mode and defocused to enable an exposure time of $40 \mathrm{~s}$. Aperture photometry was performed using an 18 pixel radius aperture and ensemble photometry using the two bright, non-variable comparison stars within the field

\footnotetext{
15 http://nsted.ipac.caltech.edu

$16 \mathrm{http} / / /$ lcogt.net/en/network/instrumentation/merope-llc-merope
}

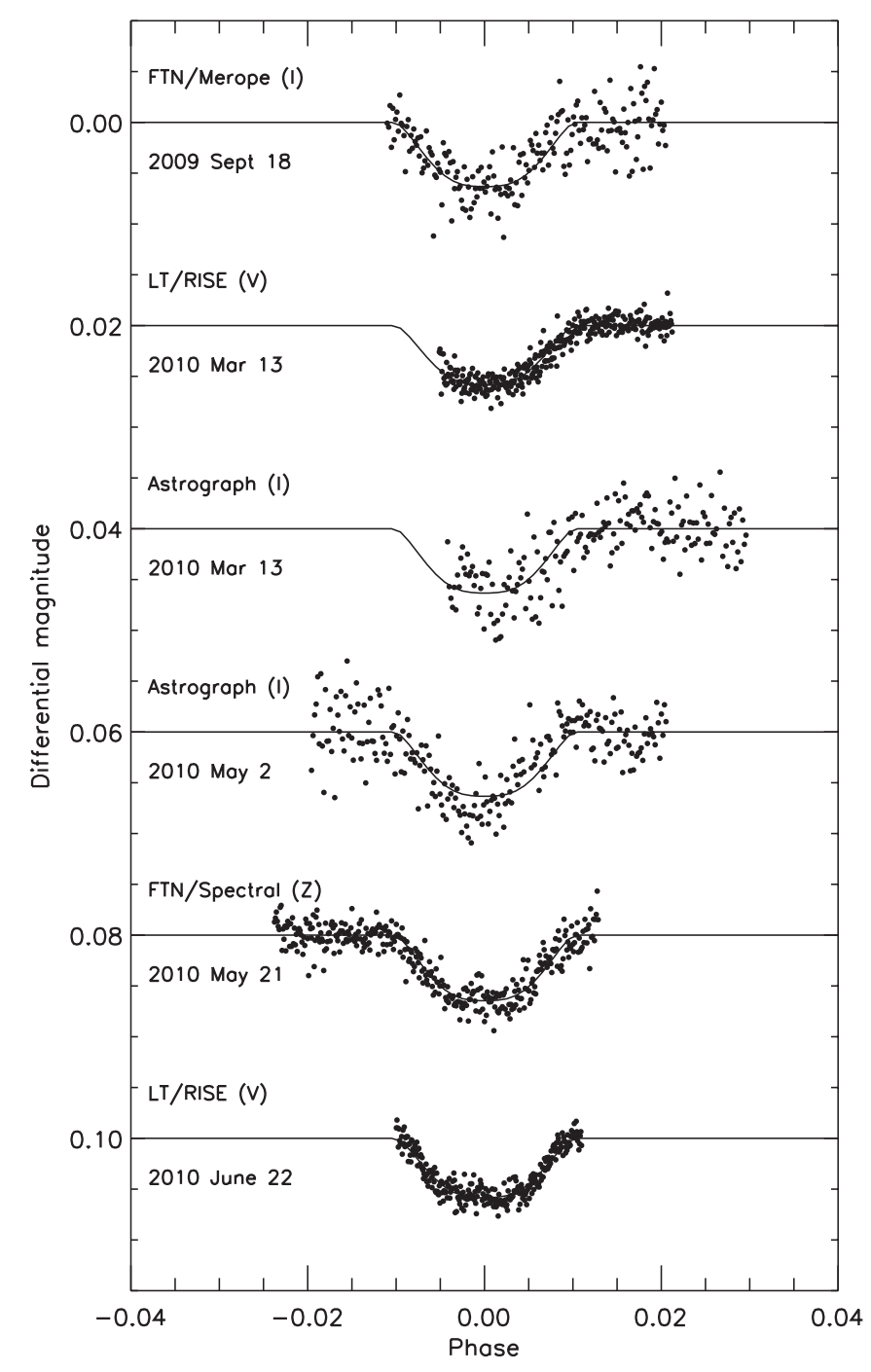

Figure 2. Photometry of transit events of HAT-P-14b taken with FTN, Astrograph, and LT in various bands. The light curves have been offset from zero by arbitrary amounts for clarity. Superimposed are the model transit light curves based on the determined system parameters (see Section 3.2).

(FITS files for this figure are available in the online journal.)

of view. By 2010 May 21, the new Spectral camera $(\mathrm{FS} 03)^{17}$ was available at FTN and defocused to obtain $20 \mathrm{~s}$ exposures. The $10.36 \times 10.22$ arcmin field of view included four bright comparison stars. These observations took advantage of the $4 \mathrm{ag}$ autoguider to maintain the locations of the stars to within an rms $\sim 2$ pixel of the same location throughout the observations and led to a noticeable improvement in the photometric precision. All the FTN data were reduced using the pipeline ${ }^{18}$ written at Liverpool John Moores University, and photometry performed using the IRAF/DAOPHOT package.

A partial transit on 2010 March 13 was observed simultaneously using the RISE instrument (Gibson et al. 2008; Steele et al. 2008) mounted on the robotic $2.0 \mathrm{~m}$ Liverpool Telescope (LT) and an Andor CCD detector on an $18 \mathrm{~cm}$ Takahashi Astrograph telescope, both located on La Palma. Two further full transits were observed; on 2010 May 2 using the Astrograph, and on 2010 June 22 by the LT.

\footnotetext{
$\overline{17}$ http://lcogt.net/en/network/instrumentation/spectral

$18 \mathrm{http} / / /$ telescope.livjm.ac.uk/Info/TelInst/Pipelines
} 
The Andor $1 \times 1 \mathrm{~K} \mathrm{e} 2 \mathrm{v}$ CCD detector has a $5 \mathrm{~s}$ readout time. For the first transit, the Astrograph was defocused with $\mathrm{FWHM}=20^{\prime \prime}$ and 685 exposures of duration $20 \mathrm{~s}$ were obtained in the $4.8 \mathrm{hr}$ period. For the second transit, the exposure time was increased to $30 \mathrm{~s}$ and FWHM $=28^{\prime \prime}$ giving 456 observations in the $4.5 \mathrm{hr}$ period. The data were reduced and differential photometry performed using the PYRAF/DAOPHOT package. The observations were subsequently binned with 3 and 2 points per bin, respectively.

The LT was defocused by $-1.2 \mathrm{~mm}$ for both transit observations using the RISE instrument, giving FWHM $=18$ and 21 pixels on the respective nights $\left(0.54 \operatorname{arcsec}_{\text {pixel }}{ }^{-1}\right)$. The CCD was binned $2 \times 2$ and exposures of $3.7 \mathrm{~s}$ taken with effectively no dead time. During the $3.0 \mathrm{hr}$ and $2.3 \mathrm{hr}$ periods, 3520 and 3290 frames were taken, respectively. The data were reduced and differential photometry performed using the ULTRACAM pipeline (Dhillon et al. 2007), and then binned with 10 points per bin.

\subsection{Spectroscopic Observations}

We obtained follow-up spectroscopic observations to determine the planetary, orbital, and stellar parameters. The star was initially observed using the FIbre-fed Echelle Spectrograph (FIES) mounted on the $2.5 \mathrm{~m}$ NOT. In total, 24 usable spectra were obtained between 2009 July 12 and 2010 April 5. Two spectra were removed from the analysis due to Moon contamination affecting the radial velocity determination. FIES was used in medium-resolution mode $(R=46,000)$ with simultaneous ThAr calibration, and observations were conducted using exposure times of $1200 \mathrm{~s}$. We used the bespoke data reduction package FIEStool ${ }^{19}$ to extract the spectra. An IDL crosscorrelation routine was used to obtain radial velocities (RVs) by fitting Gaussians to the cross-correlation functions (CCFs) of 30 spectral orders and taking the mean. A template spectrum was constructed by shifting and coadding the spectra, against which the individual spectra were cross-correlated to obtain the final velocities. The template was cross-correlated with a high signalto-noise spectrum of the Sun to obtain the absolute velocity to which the relative RVs were shifted. The RV uncertainty is given by $\sigma=\operatorname{rms}(v) / \sqrt{N}$, where $v$ is the RV of the individual orders and $N$ is the number of orders. Line bisector spans ( $\left.V_{\text {span }}\right)$ were computed using the difference in the position of the midpoint of the $\mathrm{CCF}$ at $25 \%$ and $75 \%$ of its depth.

A further nine spectra were taken between 2009 August 24 and 2009 September 5 with the stabilized echelle spectrograph SOPHIE at the $1.93 \mathrm{~m}$ telescope of Observatiore de Haute-Provence (Perruchot et al. 2008; Bouchy et al. 2009). The spectrograph was used in high efficiency mode (resolution $R=$ $40,000)$ and the observations were taken with a signal-to-noise ratio $(\mathrm{S} / \mathrm{N}) \sim 30$ to minimize the Charge Transfer Inefficiency (CTI) effect (Bouchy et al. 2009). Two 3 arcsec diameter optical fibers were used, the first centered on the target and the second on the sky to simultaneously measure the background to remove contamination from scattered moonlight. The spectra were extracted using the SOPHIE pipeline (Perruchot et al. 2008) and RVs computed from a weighted cross-correlation of each spectrum with a numerical mask of G2 spectral type, as described by Baranne et al. (1996) and Pepe et al. (2002). Cross-correlation with other masks (F0, K5, and M5) produced a consistent RV semi-amplitude, indicating that the variation is unlikely to be caused by a blended eclipsing binary system of unequal masses.

\footnotetext{
19 http://www.not.iac.es/instruments/fies/fiestool/FIEStool.html
}
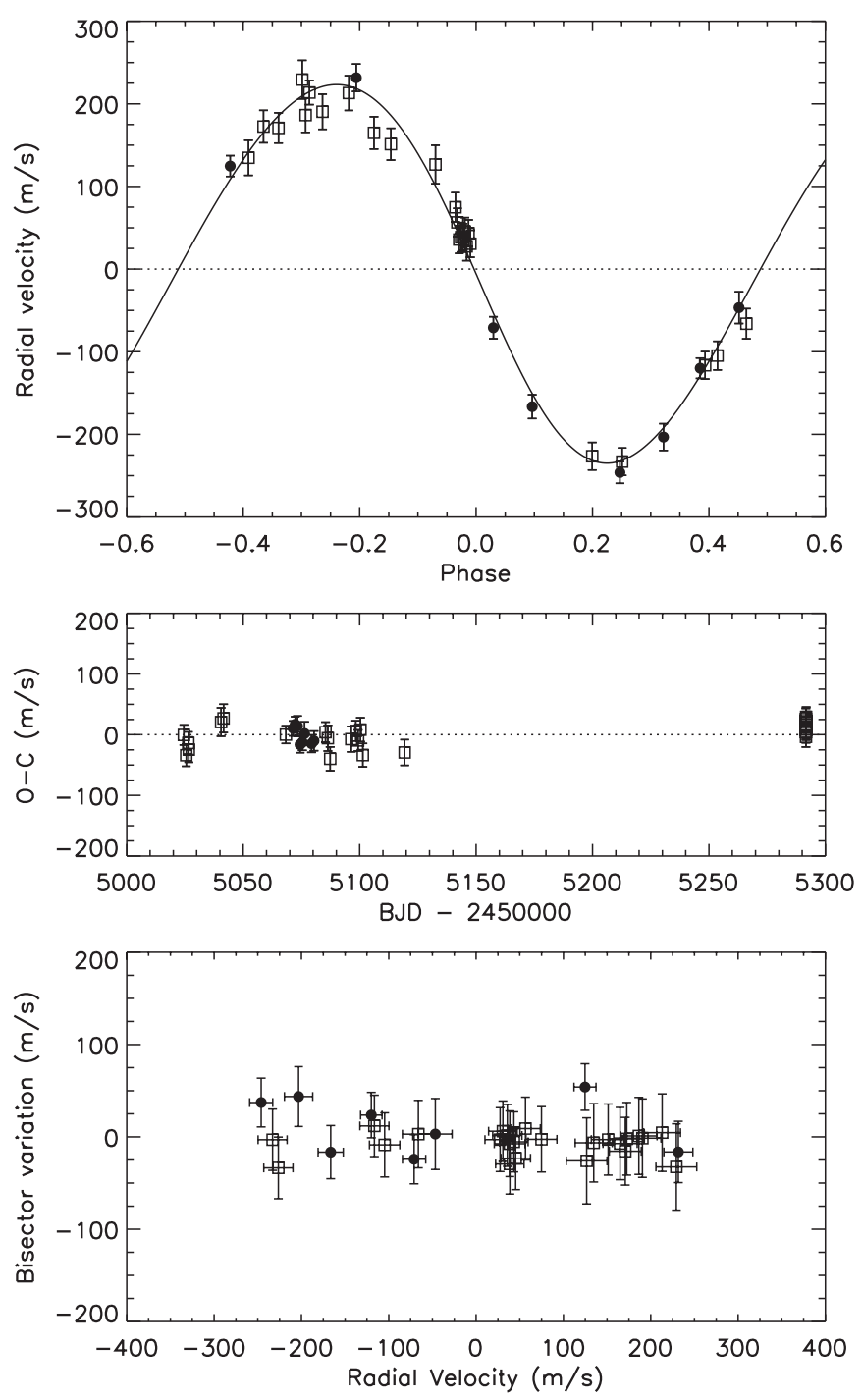

Figure 3. Upper panel: phase folded radial velocity measurements of HAT-P-14, combining data from NOT/FIES (open squares) and OHP/SOPHIE (filled circles), and superimposed with the best-fit model RV curve based on the determined system parameters. The systematic velocity for each data set was subtracted from the RVs. Middle panel: residuals from the orbital fit plotted against time. No long-term trend is visible. Lower panel: the bisector span measurements as a function of radial velocity, showing no correlation. The uncertainties in the bisector spans were taken as twice the RV uncertainties.

The RVs and line bisector spans are shown in Table 1 and plotted in Figure 3. A possible correlation between the bisector span and radial velocity was noted by T10 but was attributed to moonlight contamination rather than to a blended eclipsing binary. We find no correlation, supporting the signal's origin as a planetary companion rather than an astronomical false positive.

\subsection{High-resolution Imaging}

In order to search for additional close stars which may cause blends, we obtained high-resolution $H$ - and $K$-band imaging with the near-infrared camera INGRID, fed by the adaptive optics system NAOMI on the $4.2 \mathrm{~m}$ William Herschel Telescope, see Figure 4. Images were taken in two sky position angles (P.A., $0^{\circ}$ and $30^{\circ}$ ) in natural seeing of $0^{\prime \prime} .5$, and the corrected point-spread functions (PSFs) have an FWHM of 0". 12 and circular contours. No contaminating sources are detected to limiting magnitudes of $H \sim 17.1 \pm 0.25$ and $K_{\mathrm{s}} \sim 16.7 \pm 0.25$ at 
Table 1

Radial Velocities (RV) and Line Bisector Spans ( $\left.V_{\text {span }}\right)$ of HAT-P-14

\begin{tabular}{|c|c|c|c|c|}
\hline $\begin{array}{c}\text { BJD } \\
-2,450,000.0\end{array}$ & $\begin{array}{c}\mathrm{RV} \\
\left(\mathrm{km} \mathrm{s}^{-1}\right)\end{array}$ & $\begin{array}{c}\text { Error } \\
\left(\mathrm{km} \mathrm{s}^{-1}\right)\end{array}$ & $\begin{array}{c}V_{\text {span }} \\
\left(\mathrm{km} \mathrm{s}^{-1}\right)\end{array}$ & Instrument \\
\hline 5024.5363 & -20.6620 & 0.0071 & -0.0031 & FIES \\
\hline 5025.5236 & -20.4951 & 0.0104 & 0.0030 & FIES \\
\hline 5026.4324 & -20.2582 & 0.0105 & -0.0155 & FIES \\
\hline 5026.6481 & -20.2427 & 0.0145 & 0.0010 & FIES \\
\hline 5040.5041 & -20.1997 & 0.0179 & -0.0326 & FIES \\
\hline 5041.5629 & -20.3024 & 0.0178 & -0.0260 & FIES \\
\hline 5085.3553 & -20.5455 & 0.0072 & 0.0119 & FIES \\
\hline 5086.3534 & -20.2943 & 0.0150 & -0.0064 & FIES \\
\hline 5087.3509 & -20.2642 & 0.0126 & -0.0072 & FIES \\
\hline 5096.4062 & -20.2160 & 0.0147 & 0.0046 & FIES \\
\hline 5098.3406 & -20.6554 & 0.0073 & -0.0336 & FIES \\
\hline 5099.3361 & -20.5338 & 0.0088 & -0.0087 & FIES \\
\hline 5100.3558 & -20.2565 & 0.0127 & -0.0021 & FIES \\
\hline 5101.3677 & -20.2778 & 0.0121 & -0.0029 & FIES \\
\hline 5119.3355 & -20.2386 & 0.0150 & -0.0014 & FIES \\
\hline 5291.6152 & -20.3542 & 0.0094 & -0.0026 & FIES \\
\hline 5291.6300 & -20.3725 & 0.0080 & 0.0090 & FIES \\
\hline 5291.6449 & -20.3932 & 0.0075 & 0.0016 & FIES \\
\hline 5291.6597 & -20.3904 & 0.0095 & -0.0074 & FIES \\
\hline 5291.6746 & -20.3904 & 0.0063 & -0.0294 & FIES \\
\hline 5291.6895 & -20.3837 & 0.0079 & -0.0231 & FIES \\
\hline 5291.7043 & -20.4016 & 0.0086 & -0.0029 & FIES \\
\hline 5291.7192 & -20.3857 & 0.0063 & -0.0054 & FIES \\
\hline 5291.7340 & -20.3984 & 0.0065 & 0.0062 & FIES \\
\hline 5068.3272 & -20.1678 & 0.0146 & 0.0236 & SOPHIE \\
\hline 5071.4331 & -20.5014 & 0.0123 & 0.0540 & SOPHIE \\
\hline 5072.3251 & -20.2567 & 0.0126 & -0.0163 & SOPHIE \\
\hline 5073.3273 & -20.1498 & 0.0166 & -0.0242 & SOPHIE \\
\hline 5074.4166 & -20.4524 & 0.0133 & 0.0372 & SOPHIE \\
\hline 5075.4225 & -20.6275 & 0.0132 & 0.0031 & SOPHIE \\
\hline 5076.3691 & -20.4281 & 0.0192 & -0.0165 & SOPHIE \\
\hline 5079.3532 & -20.5479 & 0.0144 & 0.0437 & SOPHIE \\
\hline 5080.3964 & -20.5847 & 0.0162 & 0.0308 & SOPHIE \\
\hline
\end{tabular}

distances $>4 \times$ FWHM from the center of the corrected profile. At $1.5 \times$ FWHM the limiting magnitude is 2 mag brighter than this.

\section{RESULTS}

\subsection{Stellar Parameters}

We derived the fundamental stellar parameters of HAT-P-14 using the following techniques, and the results are shown in Table 2. First, we performed a spectral analysis using Spectroscopy Made Easy (see Valenti \& Piskunov 1996), following the method of Valenti \& Fischer (2005) and using the Kurucz model atmospheres (Kurucz et al. 1984). Individual NOT spectra were normalized and co-added to produce a single high signal-to-noise spectrum. The $\mathrm{H}_{\alpha}, \mathrm{Na}$ I D, and $\mathrm{Mg}$ I b lines were fitted simultaneously to determine the spectral parameters. This yielded the values $T_{\text {eff }}=6583 \pm 100 \mathrm{~K}$ and $\log g_{\text {spec }}=$ $4.02 \pm 0.1$, which indicate that the star is of spectral type F5V (Gray 2008). Values for microturbulence $\left(v_{\text {mic }}=0.85 \mathrm{~km} \mathrm{~s}^{-1}\right.$ ) and macroturbulence $\left(v_{\mathrm{mac}}=5.28 \mathrm{~km} \mathrm{~s}^{-1}\right)$ are taken from the prescription of Valenti \& Fischer (2005). The projected stellar rotation velocity $(v \sin i)$ was determined to be $v \sin i=8.4 \pm$ $1.0 \mathrm{~km} \mathrm{~s}^{-1}$ and the metallically $[\mathrm{M} / \mathrm{H}]=0.08 \pm 0.10$.

The mean stellar density $\left(\rho_{\star}\right)$, found from the light curve geometry, can be used as a luminosity indicator for stellar evolutionary models and often provides a stronger constraint than the value of $\log g$ from spectral analysis (Sozzetti et al.

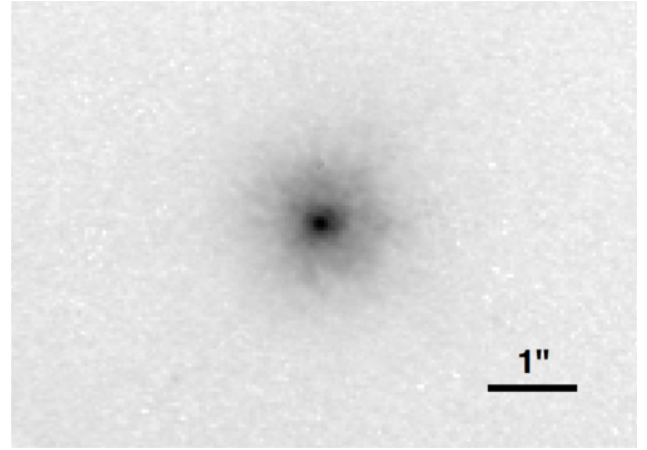

Figure 4. Adaptive optics image of HAT-P-14 in the $H$ band. The corrected PSF has an FWHM of 3 pixels $\left(00^{\prime \prime} 12\right)$. No contaminating sources are detected to a limiting magnitude of $H \sim 17.1$ mag at a distance of $>0$ '. 48 from the center of the corrected profile.

Table 2

Stellar Parameters for HAT-P-14

\begin{tabular}{|c|c|}
\hline Parameter (Unit) & Value \\
\hline \multicolumn{2}{|c|}{ Photometric and Spatial Properties } \\
\hline R.A. (J2000) & $17: 20: 27.88$ \\
\hline Decl. (J2000) & $+38: 14: 31.7$ \\
\hline$V(\mathrm{mag})$ & $9.98 \pm 0.058$ \\
\hline$J(\mathrm{mag})$ & $9.094 \pm 0.021$ \\
\hline$H(\mathrm{mag})$ & $8.927 \pm 0.020$ \\
\hline$K_{s}(\mathrm{mag})$ & $8.851 \pm 0.019$ \\
\hline$\mu_{\text {R.A. }}\left(\right.$ mas yr $\left.^{-1}\right)$ & $1.6 \pm 0.7$ \\
\hline$\mu_{\text {decl. }}\left(\right.$ mas yr $\left.^{-1}\right)$ & $4.7 \pm 0.7$ \\
\hline$U\left(\mathrm{~km} \mathrm{~s}^{-1}\right)$ & $6.5 \pm 1.0$ \\
\hline$V\left(\mathrm{~km} \mathrm{~s}^{-1}\right)$ & $-10.6 \pm 0.1$ \\
\hline$W\left(\mathrm{~km} \mathrm{~s}^{-1}\right)$ & $-6.0 \pm 0.8$ \\
\hline Galactic longitude $\left({ }^{\circ}\right)$ & 62.6 \\
\hline Galactic latitude $\left(^{\circ}\right)$ & 33.5 \\
\hline \multicolumn{2}{|c|}{ Spectroscopic Properties } \\
\hline$T_{\text {eff }}(\mathrm{K})$ & $6583 \pm 100$ \\
\hline $\log g(\mathrm{cgs})$ & $4.1 \pm 0.12$ \\
\hline$v_{\text {mic }}\left(\mathrm{km} \mathrm{s}^{-1}\right)$ & 0.85 \\
\hline$v_{\operatorname{mac}}\left(\mathrm{km} \mathrm{s}^{-1}\right)$ & 5.28 \\
\hline$v \sin i\left(\mathrm{~km} \mathrm{~s}^{-1}\right)$ & $8.4 \pm 1.0$ \\
\hline$[\mathrm{M} / \mathrm{H}]$ & $0.08 \pm 0.1$ \\
\hline \multicolumn{2}{|c|}{ Derived Properties } \\
\hline$M_{\star}\left(M_{\odot}\right)^{\mathrm{a}}$ & $1.32 \pm 0.03$ \\
\hline$R_{\star}\left(R_{\odot}\right)^{\mathrm{b}}$ & $1.53 \pm 0.10$ \\
\hline$\rho_{\star}\left(\rho_{\odot}\right)^{\mathrm{c}}$ & $0.371_{-0.058}^{+0.056}$ \\
\hline$L_{\star}\left(L_{\odot}\right)^{\mathrm{d}}$ & $3.91_{-0.37}^{+0.54}$ \\
\hline Age $(\mathrm{Gyr})^{\mathrm{d}}$ & $1.6_{-0.3}^{+0.4}$ \\
\hline Spectral type & F5V \\
\hline
\end{tabular}

Notes. The photometric and spatial properties are taken from or derived using the following sources: $V$ (TASS; Richmond et al. 2000), $J, H, K_{s}$ (2MASS; Skrutskie et al. 2006), proper motions (NOMAD; Zacharias et al. 2005), space velocities (calculated according to Johnson \& Soderblom 1987, updated to J2000, for a right-hand coordinate system and corrected for solar motion using data from Dehnen \& Binney 1998) and galactic coordinates NASA/ IPAC Extragalactic Database.

${ }^{a}$ Derived from the empirical relationship of Torres et al. (2010a).

${ }^{\mathrm{b}}$ Determined using $M_{\star}$ and $\rho_{\star}$.

${ }^{\mathrm{c}}$ Found using the light curve geometry.

${ }^{\mathrm{d}}$ From stellar models.

2007). We used a Markov Chain Monte Carlo (MCMC) approach to globally model the photometric and radial velocity data (see Section 3.2) and obtained a mean stellar density of $0.371_{-0.058}^{+0.056} \rho_{\odot}$. We then compared $T_{\text {eff }},[\mathrm{M} / \mathrm{H}]$, and $\rho_{\star}$ with the 


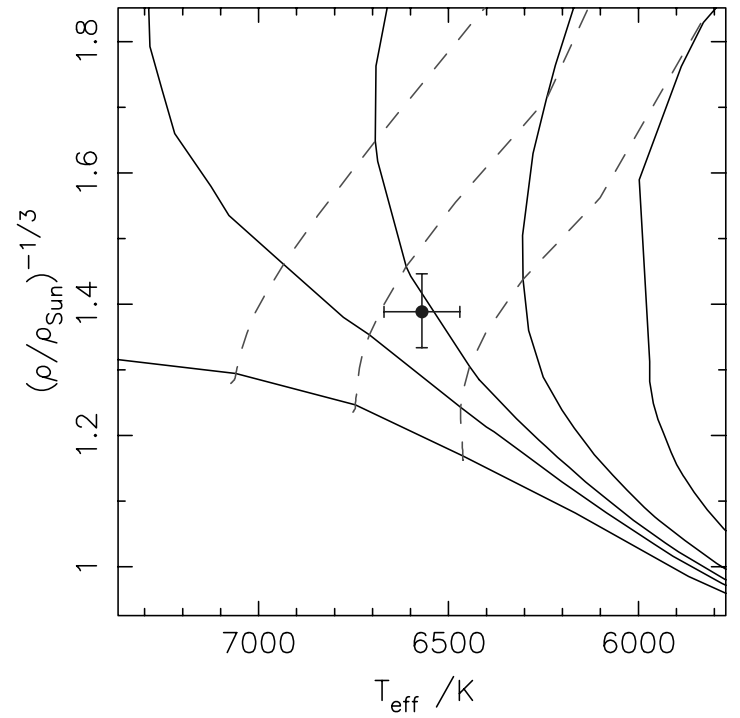

Figure 5. Theoretical stellar isochrones and mass tracks from Girardi et al. (2000) plotted as the density proxy $R_{*} / M_{*}^{1 / 3}$ against effective temperature for $[\mathrm{M} / \mathrm{H}]=0.08$. The solid lines indicate (from left to right) the ages $0.01,1,1.6$, 2.5 , and 5 Gyr and the dotted lines show masses of $1.5,1.4$, and $1.3 M_{\odot}$. The position of HAT-P-14 is indicated within the error ranges.

theoretical stellar evolutionary models of Girardi et al. (2000) to obtain the following stellar properties: $M_{\star}=1.36 \pm 0.04 M_{\odot}$, Age $=1.6_{-0.3}^{+0.4} \mathrm{Gyr}, L_{*}=3.91_{-0.37}^{+0.54} L_{\odot}$, and $\log g_{\text {iso }}=4.19 \pm$ 0.03 . The model isochrones are shown in Figure 5.

The value of $\log g$ obtained from the isochrone fit is somewhat larger than that found from spectral analysis, which was also noted by $\mathrm{T} 10$. We investigated the effect this higher $\log g$ would have on the fit to the spectral line shapes by fixing $\log g_{\text {spec }}$ to the isochrone value and reassessing the spectral analysis. We found that in order to retain a good fit to the $\mathrm{Mg} \mathrm{I} \mathrm{b}$ lines, the magnesium abundance $[\mathrm{Mg} / \mathrm{H}]$ must be reduced by 0.12 (in a similar fashion to WASP-1; see Stempels et al. 2007) due to the inherent anti-correlation between $[\mathrm{Mg} / \mathrm{H}]$ and $\log g$. The degeneracies between the spectroscopic parameters do not rule out the higher $\log g$ value as long as $[\mathrm{Mg} / \mathrm{H}]$ is allowed to be underabundant, and we take the uncertainty in $\log g$ to include both values. Altering $\log g$ and $[\mathrm{Mg} / \mathrm{H}]$ in this fashion had no significant effect on the derived effective temperature or metal abundance.

\subsection{Planet Parameters}

To determine the properties of the planet HAT-P-14b, we simultaneously modeled the light curves and RVs with a global MCMC fit. Details of this process are described in Collier Cameron et al. (2007) and Pollacco et al. (2008). The free parameters in the fit are: orbital period $P$, transit epoch $T_{0}$, transit duration $T_{\text {dur }}$, ratio of planet radius to star radius $\left(R_{\mathrm{P}} / R_{\star}\right)^{2}$, impact parameter $b$, RV semi-amplitude $K$, Lagrangian elements $e \cos \omega$ and $e \sin \omega$ where $e$ is the eccentricity and $\omega$ is the longitude of periastron, and the systematic offset velocity $\gamma$. In this particular case, two systematic velocities were fit to allow for instrumental offsets between the SOPHIE and FIES data sets. These were found to be: $\gamma_{\text {SOPHIE }}=-20.4294 \pm$ $0.0009 \mathrm{~km} \mathrm{~s}^{-1}$ and $\gamma_{\text {FIES }}=-20.3833 \pm 0.0026 \mathrm{~km} \mathrm{~s}^{-1}$. To normalize the light curves, a linear de-trending was applied and the normalization parameters were allowed to float during the fit.

The Markov chain length was 10,000 points and the resulting best-fit parameters and uncertainties are shown in Table 3. To account for red noise, the residuals of the non-WASP photometry were averaged into 6 minute bins before calculating $\chi^{2}$. The photometric error bars were re-scaled so that $\chi^{2} \sim N_{\text {bin }}$ to guard against the possibility that a densely sampled photometric data set with correlated errors should dominate the result. We also ran the routine using 12 minute binning and this gave consistent values with those derived using the 6 minute binning. The Claret (2000, 2004) nonlinear limb-darkening coefficients were chosen for the appropriate stellar temperature and photometric passband appropriate for each light curve.

In order to estimate the stellar mass and properties derived from it, we used the Torres et al. (2010a) empirical calibrations from eclipsing binaries. This method, adapted by Enoch et al. (2010), computes the stellar mass as a function of $T_{\text {eff }}, \log \rho_{*}$, and $[\mathrm{M} / \mathrm{H}]$ and has the advantage of being independent of stellar models. We find $M_{\star}=1.32 \pm 0.03 M_{\odot}$, similar to the stellar mass derived from the isochrone analysis undertaken here $\left(M_{\star}=\right.$ $1.36 \pm 0.04 M_{\odot}$, Padova models $)$ and in T10 $\left(M_{\star}=1.39 \pm\right.$ $0.05 M_{\odot}$, YY models).

We performed the MCMC fit and derived the following planetary parameters: $M_{p}=2.28 \pm 0.07 M_{\mathrm{J}}, R_{p}=1.20 \pm$ $0.15 R_{\mathrm{J}}$, and $\rho_{p}=1.32 \pm 0.37 \rho_{\mathrm{J}}$. In addition, we analyzed the data solely from T10 and found that the fitted parameters were completely consistent with their results. The results are shown in Table 3 and are in good agreement.

\subsection{Eccentricity}

In an initial fit to the SOPHIE and FIES RVs, the eccentricity was allowed to float, yielding $e=0.054 \pm 0.017$. We analyzed the data sets independently and found that a jitter term of $15 \mathrm{~m} \mathrm{~s}^{-1}$ was required to be added in quadrature to the FIES RV uncertainties in order to obtain $\chi_{\text {red }}^{2}=\chi^{2} /$ dof $=1$ (dof $=$ number of points - number of fitted parameters). This may due to astrophysical noise, as also noted by T10, and/or instrumental systematic noise which has not been accounted for. The FIES $\mathrm{RV}$ s do not significantly detect an eccentric orbit, yielding $e=$ $0.04 \pm 0.03$, but equally do not rule one out.

In contrast, the SOPHIE RVs do not require any jitter term to reconcile the rms scatter with the measured uncertainties. Fitting the SOPHIE RVs alone, a more significant detection of eccentricity was found, $e=0.081 \pm 0.022$ with $\chi^{2}=2.0$ for 9 $\mathrm{RV}$ points and 4 fitted parameters $\left(K, e \cos \omega, e \sin \omega, \gamma_{\text {SOPHIE }}\right)$. Fitting a purely circular orbit to the same data, we found $\chi_{\text {circ }}^{2}=$ 22.6. Applying the Lucy-Sweeney test (Lucy \& Sweeney 1971), the eccentricity is detected at the $3 \sigma$ level.

A joint fit to both data sets (including the FIES jitter term) reveals $e=0.066 \pm 0.023, \chi^{2}=34.4$ and $\chi_{\text {circ }}^{2}=49$ for $33 \mathrm{RV}$ points and 5 fitted parameters (including two systematic velocities, $\gamma_{\text {SOPHIE }}$ and $\gamma_{\text {FIES }}$ ), indicating the eccentricity is non-zero at the $2.7 \sigma$ level. We also apply the test to the T10 Keck RVs, including their $7.3 \mathrm{~m} \mathrm{~s}^{-1}$ jitter term. We find the eccentricity is detected at the $4 \sigma$ level, $e=0.104 \pm 0.012$, with $\chi^{2}=12.3, \chi_{\text {circ }}^{2}=83.9,14$ RVs, and 4 fitted parameters.

The difference between the eccentricity derived from the NOT/SOPHIE and Keck data suggests that the uncertainties may have been underestimated. It has been shown that determining the significance and uncertainties of orbital eccentricity from sparse data is difficult, and there had been several initial detections which are now thought to be spurious, e.g., WASP-12 (Hebb et al. 2009; Husnoo et al. 2010; Campo et al. 2011); WASP-10 (Christian et al. 2009; Maciejewski et al. 2010). The FIES data are not regularly spaced enough for eccentricity measurements and the constraints mostly rely on the SOPHIE 
Table 3

System Parameters for HAT-P-14b

\begin{tabular}{|c|c|c|}
\hline Parameter (Unit) & This Paper & Torres et al. (2010b) \\
\hline \multicolumn{3}{|c|}{ Photometric Parameters } \\
\hline$P$ (days) & $4.627649 \pm 0.000008$ & $4.627669 \pm 0.000005$ \\
\hline$T_{0}(\mathrm{HJD})$ & $2455310.29018 \pm 0.00048$ & $2455134.43854 \pm 0.00026$ \\
\hline$T_{\text {dur }}$ (days) & $0.0925 \pm 0.0021$ & $0.0912 \pm 0.0017$ \\
\hline$\Delta F=R_{\mathrm{P}}^{2} / R_{*}^{2}$ & $0.00629 \pm 0.00016$ & $0.00648 \pm 0.00024$ \\
\hline$R_{\mathrm{P}} / R_{*}$ & $0.0793 \pm 0.0010$ & $0.0805 \pm 0.0015$ \\
\hline$b$ & $0.924_{-0.009}^{+0.016}$ & $0.891_{-0.008}^{+0.007}$ \\
\hline$a / R_{*}$ & $8.40 \pm 0.54$ & $8.87 \pm 0.29$ \\
\hline$i\left(^{\circ}\right)$ & $83.2 \pm 0.6$ & $83.5 \pm 0.3$ \\
\hline \multicolumn{3}{|c|}{ Spectroscopic Parameters } \\
\hline$K\left(\mathrm{~km} \mathrm{~s}^{-1}\right)$ & $0.2293 \pm 0.0050$ & $0.2190 \pm 0.0033$ \\
\hline$e \cos \omega$ & $-0.025 \pm 0.011$ & $-0.009 \pm 0.009$ \\
\hline$e \sin \omega$ & $0.061 \pm 0.026$ & $0.106 \pm 0.013$ \\
\hline$e$ & $0.066 \pm 0.023$ & $0.107 \pm 0.013$ \\
\hline$\omega\left(^{\circ}\right)$ & $111_{-9}^{+16}$ & $94 \pm 4$ \\
\hline \multicolumn{3}{|c|}{ Derived Parameters } \\
\hline$M_{\mathrm{P}}\left(M_{\mathrm{Jup}}\right)$ & $2.28 \pm 0.07$ & $2.23 \pm 0.06$ \\
\hline$R_{\mathrm{P}}\left(R_{\mathrm{Jup}}\right)$ & $1.20 \pm 0.15$ & $1.15 \pm 0.05$ \\
\hline$\rho_{\mathrm{P}}\left(\rho_{\mathrm{J}}\right)$ & $1.32 \pm 0.37$ & $1.47 \pm 0.20$ \\
\hline $\log g_{P}(\mathrm{cgs})$ & $3.56 \pm 0.09$ & $3.62 \pm 0.04$ \\
\hline$a(\mathrm{AU})$ & $0.0596 \pm 0.0005$ & $0.0606 \pm 0.0007$ \\
\hline$T_{\mathrm{eq}, \mathrm{A}=0}(\mathrm{~K})$ & $1612 \pm 50$ & $1570 \pm 34$ \\
\hline
\end{tabular}

data. In fact, the SOPHIE value alone is more consistent with the Keck value.

The fact that for the SOPHIE data, $\chi_{\text {red }}^{2}$ is near one around an eccentric-orbit fit can either mean that the eccentricity measurement is correct and there are no unrecognized sources of residuals, or that the eccentricity is different and the error budget is underestimated. Although care has been taken to keep the $\mathrm{S} / \mathrm{N}$ constant to avoid the CTI effect, the values of the RV uncertainties vary and zero-point shifts could be present which may bias the eccentricity measurement.

\section{CONCLUSIONS}

We report the independent discovery and follow-up observations of the transiting planet HAT-P-14b found by T10. We have analyzed the follow-up data we used to secure the detection, and derived an independent set of planetary parameters which are consistent with those found by T10. We provide additional evidence, through adaptive optics observations and additional bisector span measurements, that the planetary signal is not caused by astronomical false positives.

We find the properties of the parent star to be in good agreement with those found by $\mathrm{T} 10$, which is reassuring given the different techniques used in their derivation. The star is a hot F5 dwarf with $T_{\text {eff }}=6583 \pm 100 \mathrm{~K}$ and appears to be relatively young at $1.6_{-0.3}^{+0.4} \mathrm{Gyr}$, with a slightly super-solar metallicity $[\mathrm{M} / \mathrm{H}]=0.08$. Using the empirical calibrations of Torres et al. (2010a) we determine the stellar mass to be $1.32 \pm 0.03 M_{\odot}$, which agrees well with the values we derive from theoretical stellar models.

We find that HAT-P-14b has $R_{p}=1.20 \pm 0.15 R_{\mathrm{J}}, M_{p}=$ $2.28 \pm 0.07 M_{\mathrm{J}}$, and $\rho_{p}=1.32 \pm 0.37 \rho_{\mathrm{J}}$. It appears to blur the bi-modal distribution noted by Bakos et al. (2010) in which massive $\left(M_{p} \sim 2 M_{\mathrm{J}}\right)$ planets appear either inflated $\left(R_{p} \sim 1.3 R_{\mathrm{J}}\right)$ or compact $\left(R_{p} \sim 1 R_{\mathrm{J}}\right)$. We find the orbit to be slightly eccentric, $e=0.066 \pm 0.023$, which hints at the possibility of another body in the system.
As noted by T10, the system promises excellent opportunities for further follow-up and characterization, in particular due to the brightness of the host star, $V_{\mathrm{mag}}=9.98$. The star is relatively fast rotating for a planet host, $v \sin i=8.4 \pm 1.0 \mathrm{~km} \mathrm{~s}^{-1}$, making it a good candidate for the detection of the Rossiter-McLaughlin (RM) effect (Rossiter 1924; McLaughlin 1924). However, due to the very high impact parameter, $b \sim 0.9$, the RM amplitude is only expected to be $\sim 20 \mathrm{~m} \mathrm{~s}^{-1}$. Despite this, it is still an attractive target as Winn et al. (2010) and Schlaufman (2010) have shown that misaligned planets appear to preferentially orbit stars with high $T_{\text {eff }}$ and $M_{\star}$ such as HAT-P-14. ${ }^{20}$

The high temperature of the host star places HAT-P-14b in the highly irradiated "pM" category of Fortney et al. (2008) and is a good candidate for investigating temperature inversions and day/night contrasts. An observation of the secondary transit would be particularly interesting to further constrain the low eccentricity. However, the orbital geometry of the system means that HAT-P-14b is on the borderline of having no secondary eclipse. Using the Keck eccentricity, the analysis predicts that it will not occur with more than $3 \sigma$ confidence. However, the fit to the data presented in this paper alone suggests that the secondary eclipse could be grazing. Since these opposing predictions arise from extremely small differences in orbital eccentricity, whether or not we observe a secondary eclipse will provide further constraints on the system geometry. HAT-P-14 thus provides excellent opportunities for future investigations and informative characterization.

The SuperWASP Consortium consists of astronomers primarily from Queen's University Belfast, St. Andrews, Keele, Leicester, The Open University, Isaac Newton Group La Palma, and Instituto de Astrofísica de Canarias. The SuperWASP-N camera is hosted by the Isaac Newton Group on La Palma and we are grateful for their continuing support and assistance. Funding for WASP comes from consortium universities

\footnotetext{
20 After this paper was submitted for publication, the orbit of HAT-P-14b was indeed observed to be misaligned (Winn et al. 2011).
} 
and from the UK's Science and Technology Facilities Council. Based on observations made at Observatoire de Haute Provence (CNRS), France and at the Nordic Optical Telescope, operated on the island of La Palma jointly by Denmark, Finland, Iceland, Norway, and Sweden, in the Spanish Observatorio del Roque de los Muchachos of the Instituto de Astrofísica de Canarias. Also based on observations made with the William Herschel Telescope operated on the island of La Palma by the Isaac Newton Group in the Spanish Observatorio del Roque de los Muchachos of the Instituto de Astrofísica de Canarias. The Liverpool Telescope is operated on the island of La Palma by Liverpool John Moores University in the Spanish Observatorio del Roque de los Muchachos of the Instituto de Astrofísica de Canarias with financial support from the UK Science and Technology Facilities Council. We thank Tom Marsh for the use of the ULTRACAM pipeline. F.P.K. is grateful to AWE Aldermaston for the award of a William Penny Fellowship. E.K.S. thanks R. M. Crockett for his thorough proofreading and excellent image-making.

\section{REFERENCES}

Alonso, R., et al. 2004, ApJ, 613, L153

Baglin, A., et al. 2006, in COSPAR Scientific Assembly, Vol. 36, COSPAR Plenary Meeting, 3749

Bakos, G. Á., Lázár, J., Papp, I., Sári, P., \& Green, E. M. 2002, PASP, 114 974

Bakos, G. Á., et al. 2009, ApJ, 696, 1950

Bakos, G. Á., et al. 2010, arXiv:1008.3388

Baranne, A., et al. 1996, A\&AS, 119, 373

Borucki, W. J., et al. 2010, Science, 327, 977

Bouchy, F., et al. 2009, A\&A, 505, 853

Burke, C. J., et al. 2008, ApJ, 686, 1331

Campo, C. J., et al. 2011, ApJ, 727, 125

Christian, D. J., et al. 2009, MNRAS, 392, 1585

Claret, A. 2000, A\&A, 363, 1081

Claret, A. 2004, A\&A, 428, 1001

Collier Cameron, A., et al. 2006, MNRAS, 373, 799

Collier Cameron, A., et al. 2007, MNRAS, 375, 951

Dehnen, W., \& Binney, J. J. 1998, MNRAS, 298, 387

Dhillon, V. S., et al. 2007, MNRAS, 378, 825
Enoch, B., Collier Cameron, A., Parley, N. R., \& Hebb, L. 2010, A\&A, 516, A33

Fabrycky, D. C., \& Winn, J. N. 2009, ApJ, 696, 1230

Fortney, J. J., Lodders, K., Marley, M. S., \& Freedman, R. S. 2008, ApJ, 678, 1419

Gibson, N. P., et al. 2008, A\&A, 492, 603

Girardi, L., Bressan, A., Bertelli, G., \& Chiosi, C. 2000, A\&AS, 141, 371

Gray, D. F. 2008, The Observation and Analysis of Stellar Photospheres (Cambridge: Cambridge Univ. Press)

Hebb, L., et al. 2009, ApJ, 693, 1920

Husnoo, N., et al. 2010, arXiv:1004.1809

Johnson, D. R. H., \& Soderblom, D. R. 1987, AJ, 93, 864

Kurucz, R. L., Furenlid, I., Brault, J., \& Testerman, L. 1984, Solar Flux Atlas from 296 to $1300 \mathrm{~nm}$ (Sunspot, NM: National Solar Observatory)

Lucy, L. B., \& Sweeney, M. A. 1971, AJ, 76, 544

Maciejewski, G., et al. 2010, MNRAS, 407, 2625

McCullough, P. R., Stys, J. E., Valenti, J. A., Fleming, S. W., Janes, K. A., \& Heasley, J. N. 2005, PASP, 117, 783

McLaughlin, D. B. 1924, ApJ, 60, 22

Pál, A., et al. 2009, ApJ, 700, 783

Pepe, F., et al. 2002, A\&A, 388, 632

Perruchot, S., et al. 2008, Proc. SPIE, 7014, 17

Pollacco, D. L., et al. 2006, PASP, 118, 1407

Pollacco, D., et al. 2008, MNRAS, 385, 1576

Richmond, M. W., et al. 2000, PASP, 112, 397

Rossiter, R. A. 1924, ApJ, 60, 15

Schlaufman, K. C. 2010, ApJ, 719, 602

Skrutskie, M. F., et al. 2006, AJ, 131, 1163

Southworth, J. 2010, MNRAS, 408, 1689

Sozzetti, A., Torres, G., Charbonneau, D., Latham, D. W., Holman, M. J., Winn, J. N., Laird, J. B., \& O'Donovan, F. T. 2007, ApJ, 664, 1190

Steele, I. A., Bates, S. D., Gibson, N., Keenan, F., Meaburn, J., Mottram, C. J., Pollacco, D., \& Todd, I. 2008, Proc. SPIE, 7014, 217

Stempels, H. C., Collier Cameron, A., Hebb, L., Smalley, B., \& Frandsen, S. 2007, MNRAS, 379, 773

Torres, G., Andersen, J., \& Giménez, A. 2010a, A\&AR, 18, 67

Torres, G., et al. 2010b, ApJ, 715, 458

Triaud, A. H. M. J., et al. 2010, A\&A, 524, A25

Valenti, J. A., \& Fischer, D. A. 2005, ApJS, 159, 141

Valenti, J. A., \& Piskunov, N. 1996, A\&AS, 118, 595

West, R. G., et al. 2009, A\&A, 502, 395

Winn, J. N., Fabrycky, D., Albrecht, S., \& Johnson, J. A. 2010, ApJ, 718, L145

Winn, J. N., et al. 2011, AJ, 141, 63

Zacharias, N., Monet, D. G., Levine, S. E., Urban, S. E., Gaume, R., \& Wycoff, G. L. 2005, VizieR Online Data Catalog, 1297, 0 